\title{
Central Nervous System Neuroblastoma
}

National Cancer Institute

\section{Source}

National Cancer Institute. Central Nervous System Neuroblastoma. NCI Thesaurus.

Code C4826.

A neuroblastoma arising from the cerebral hemispheres. 